\title{
Enzymatic action of proteases and chitinases of the nematophagous fungi Pochonia chlamydosporia on Dioctophyma renale eggs
}

\section{Angélica de Souza Gouveia ${ }^{1 *}$, Filippe Elias de Freitas Soares ${ }^{1}$, Fabio Ribeiro Braga ${ }^{2,3}$, Alexandre de Oliveira Tavela², Juliana Milani Araújo², Leandro Abreu da Fonseca ${ }^{3}$, Jackson Victor de Araújo², José Humberto de Queiróz ${ }^{1}$}

\author{
${ }^{I}$ Departamento de Bioquímica e Biologia Molecular. Universidade Federal de Viçosa, Viçosa/MG \\ ${ }^{2}$ Departamento de Veterinária, Universidade Federal de Viçosa, Viçosa/MG \\ ${ }^{3}$ Universidade Vila-Velha, Vila Velha/ES \\ (*) Corresponding author: E-mail: angelica.s.gouveia@gmail.com
}

\section{Abstract}

The giant kidney worm, Dioctophyma renale, is the largest nematode known. Eggs of $D$. renale are eliminated through urine of the dog and its development occurs in the external environment. In addition, its shell thick and wrinkled confers resistance in the environment for years. However, measures such as biological control that can help decrease egg concentrations may aid in the control of this zoonosis. The present study aimed to evaluate the enzymatic action of proteases and chitinases produced by Pochonia chlamydosporia(VC4) on Dioctophymarenale eggs. Four groups were formed in sterile tubes, three treatment groups, one group treated with only $\mathrm{VC} 4$ proteases, one group treated with only VC4 chitinases, one group treated with both classes of enzymes (proteases and chitinases) and one control group (no enzyme). To avoid protease activity in the group treated only with chitinases, the protease inhibitor PMSF (phenylmethylsulfonyl fluoride) was used. One hundred $D$. renale eggs were transferred into sterile tubes containing enzyme for each treated group. The control group consisted of one hundred $D$. renale eggs. The sterile tubes were incubated at $28{ }^{\circ} \mathrm{C}$ in the dark for 24 hours. After 24 hours, the total number of $D$. renale intact eggs present in each of the treated and control tubes was calculated. At the end of the experiment, it was observed that the proteases and chitinases of $P$. chlamydosporia (VC4), either individually or together, have caused a significant percentage reduction $(\mathrm{p}<0.01)$ on the number of viable eggs 
of $D$. renale, compared to control, with the following reduction values: $27.8 \%$ (proteases), 29.4\% (chitinases) and 43.4\% (proteases + chitinases). Thus, the constant search for alternatives that may help combat the various infectious forms (eggs and/or larvae) of potentially zoonotic nematodes are important, as the use of enzymes from fungi destroyers of eggs.

Keywords: ovicidal fungus, dioctophymosis, protease, chitinase,biological control

Apoio financeiro: CNPq, FAPEMIG, CAPES. 